\title{
TRAYECTORIAS EN CLAVE TRANSNACIONAL. GENERACIÓN, JUVENTUD(ES), EDUCACIÓN Y FAMILIA EN LA DEFINICIÓN DE LOS PROYECTOS MIGRATORIOS DE HAITIANOS Y HAITIANAS HACIA LA ARGENTINA
}

\author{
Transnational key trajectories. \\ Generation, youth(s), education and family in the definition \\ of Haitian migratory projects towards Argentina
}

Carina Trabalón*

\begin{abstract}
Resumen. En el presente trabajo se aborda la definición de los proyectos migratorios de haitianos y haitianas hacia las ciudades de Córdoba y Rosario, Argentina, durante el periodo 2007-2019. Mediante un análisis de sus trayectorias en clave transnacional se busca problematizar algunos de los enunciados más recurrentes referidos a los migrantes haitianos y haitianas en Argentina, por ejemplo, aquellos vinculados a la pobreza, el terremoto ocurrido en 2010 y el modo en que se entiende su relación con la diáspora haitiana en el mundo. Desde un enfoque metodológico cualitativo, basado en la realización de entrevistas en profundidad y observación participante en diferentes eventos y espacios sociales, se busca oponer a ciertas miradas victimizantes, la definición de proyectos migratorios construidos generacional y transnacionalmente a partir de estrategias de reproducción familiar específicas que tienen lugar en el marco de una apuesta por la educación ligada a su condición de jóvenes.
\end{abstract}

Palabras clave: trayectorias; transnacionalismo; migración haitiana; juventudes; generación.

Abstract. This work deals with the definition of haitian migration projects
towards the cities of Córdoba and Rosario, Argentina, during the period
$2007-2019$. Through an analysis of their trajectories in a transnational
key, it seeks to problematize some of the most recurrent statements
referring to Haitian migrants in Argentina, for example, those related to
poverty, the earthquake that occurred in 2010 and the way in which their
relationship with the Haitian diaspora in the world. Thus, from a qualitative
methodological approach, based on in-depth interviews and participant
observation in different events and social spaces, it seeks to oppose certain
victimized views, the definition of migration projects built generationally

Universidad Nacional de Villa María. Cordoba, Argentina. E-mail: carinatrabalon522@gmail. com. Orcid: https://orcid.org/: 0000-0003-3675-2338. 
and transnationally from specific family reproduction strategies, which take place within the framework of a commitment to education linked to their youth status.

Keywords: trajectories; transnationalism; haitian migration; youth; generation.

\section{Introducción}

Diferentes estudios sobre migración y transnacionalismo familiar han destacado la importancia de incorporar la dinámica familiar en el análisis de los procesos migratorios, contraponiéndose a aquella inclinación a considerar la migración "como un asunto de varones, resultado de decisiones y acciones individuales" (Gil Araujo, Pedone, 2014). Como señalan estas autoras, esto resulta particularmente evidente en el cruce de género y transnacionalismo, es decir, en el caso de las mujeres que migran y sus implicancias en la organización familiar y el cuidado de sus hijos e hijas. Este señalamiento constituye un buen punto de partida para pensar el vínculo entre migración y juventudes en la medida que permite advertir el relieve que adquiere la familia en la definición y desarrollo de los proyectos migratorios, en este caso, de los jóvenes que migran, cuyas experiencias plantean puntos en común pero también diferencias significativas con relación a los "jóvenes de origen migrante" o "jóvenes hijos de migrantes" a quienes la literatura les ha dedicado la mayor atención ${ }^{1}$. En este trabajo en particular, se aborda la definición de los proyectos migratorios a partir del cruce entre generación, juventud, familia y educación. Para ello, se propone un abordaje a partir de la articulación entre la noción de trayectorias de Sayad (2010) y algunos aportes de la perspectiva transnacional (Feldman-Bianco, 2015, 2018; Glick-Schiler, 2010; Levitt, GlickSchiller, 2004; Suárez Navaz, 2006).

Como se verá, la noción de trayectorias permite incluir el recorrido temporal y espacial de los sujetos y mostrar las diferentes configuraciones sociales a través de las cuales se llevan adelante proyectos migratorios históricamente situados. De acuerdo con Sayad (2010),

En lugar de dedicarse a explicar la situación de los emigrados (en realidad, de los inmigrados), única y exclusivamente, por la historia de su estancia en Francia, hay que tomar por objeto la relación entre el sistema de disposiciones de los emigrados y el conjunto de mecanismos a los que están sometidos como efecto de la emigración. No se puede comprender plenamente esta relación más que

1 En Argentina, distintas investigaciones vienen trabajando el nexo migración y juventud(es) desde diferentes dimensiones y abordajes entre los que se destacan las temáticas centradas en las experiencias formativas/educativas de hijos de migrantes, el cruce entre jóvenes, generación y participación política y, las trayectorias laborales (Novaro, 2016, 2019; Beheran, 2009; Gavazzo 2013, 2018; Rosas, 2014; Miranda, Cravino, Garro, 2012; Maggi, Hendel, 2019; Suaréz, 2019; Groisman, 2019; Zenklusen, 2020; Trpin, 2007; entre otros). 
a condición de interrogarse acerca de los procesos diferenciados que los han llevado a su posición actual y cuyo origen se debe buscar fuera de la emigración. Únicamente las trayectorias emigrantes reconstituidas íntegramente pueden dar cuenta del sistema completo de determinaciones que, habiendo actuado antes de la emigración y, siguiendo actuando, con una forma modificada, durante la inmigración, han llevado al emigrado a la actual situación. (Sayad, 2010, p. 57)

De acuerdo con este autor, la migración se configura al mismo tiempo como emigración e inmigración ${ }^{2}$, por lo tanto, antes de ser inmigrante se es emigrante y, por ello, "una sociología de la migración debe comenzar, no desde la sociedad receptora, sino desde la estructura y las contradicciones de las comunidades emisoras" (Bourdieu, Wacquant, 2000, p.174, traducción propia). En la medida en que las trayectorias remiten a las posiciones sociales sucesivamente ocupadas y que estas posiciones sociales son relacionales, la noción permite destacar tanto la dimensión subjetiva de procesos migratorios como sus condicionantes estructurales. De este modo, las trayectorias incluyen los movimientos geográficos pero también los sobrepasan, destacando la necesidad de pensarlos en marcos temporales y espaciales más amplios en los que entran en consideración las características sociales de los sujetos, es decir, las disposiciones socialmente determinadas que caracterizan a los migrantes antes de migrar así como, más tarde, la reconfiguración de capitales y posiciones que se produce a lo largo del tiempo en el desarrollo de sus vidas en la "sociedad receptora" (Sayad, 2010).

Por otra parte, aunque la noción de trayectorias contribuye de manera destacada a forjar una comprensión más adecuada de las prácticas de los migrantes desde el punto de vista sociológico, es necesario pensarla complementariamente con algunos aportes de la perspectiva transnacional. En esta línea, la dimensión transnacional de las trayectorias permite situar las estrategias de los sujetos en conexión con las redes de relaciones que conectan diferentes espacios, escalas y temporalidades (Feldman-Bianco, 2015, 2018). De tal forma, se considera no solo la relación inmigración-emigración y sus condiciones sociales específicas de producción sino también la simultaneidad entre diferentes espacios y temporalidades a través de múltiples redes de relaciones que remiten a procesos sociales e históricos que van más allá de las fronteras nacionales de los respectivos Estados de "origen", "destino" así como otros posibles países involucrados en la situación de movilidad según los casos específicos (Levitt, Glick-Schiller, 2004). Estas consideraciones permiten incluir el estudio de las trayectorias en un complejo de redes que conecta a las personas a través de las fronteras de los estados nacionales y

\footnotetext{
2 Varios aspectos de esta dimensión han sido analizados y recuperados por diferentes estudiosos de las migraciones (Gil Araujo, 2010; Domenech, 2019; Jimenez Zunino, 2018; Avallone, Santamaría, 2018, entre otros) aunque no en todos los casos específicamente con relación a noción de trayectorias.
} 
las localidades específicas (Feldman-Bianco, 2015, 2018; Glick-Schiller, 2010; Suárez Navaz, 2006). A través de la reformulación de la relación entre espacio social y fronteras, el transnacionalismo contribuye a hacer visibles formas de vida y estrategias de los sujetos migrantes destacando la importancia que comportan las relaciones de poder, mediadas por la clase, el género y la raza, en la conformación de los movimientos migratorios a través de las fronteras.

En este cuadro, la noción de proyecto migratorio vinculado a las trayectorias remite a las ideas que tienen los y las jóvenes sobre por qué migraron, es decir, está asociada al posicionamiento subjetivo frente a la situación de movilidad: "lo que ocurre en la cabeza del migrante mientras su cuerpo se desplaza en el espacio-tiempo, durante los meses previos a ese desplazamiento y los años siguientes a él" (García Borrego, 2007, p. 162). En este caso, se analiza la instancia de definición del proyecto migratorio a partir de la relevancia y el lugar que adquieren los procesos sociales vinculados a la juventud, la generación y la diáspora en el desarrollo de la vida de estos y estas jóvenes. Para ello, el trabajo se organiza en tres apartados que remiten a: en primer lugar, las condiciones socio-históricas de emergencia del proyecto migratorio; en segundo lugar, la apuesta familiar y generacional por la educación asociada a su condición de jóvenes y; en tercer lugar, su (re) configuración como estrategia de reproducción familiar y transnacional.

En esta dirección, se subraya la importancia que supone, en términos analíticos, realizar un abordaje de los proyectos migratorios desde la noción de trayectorias en clave transnacional puesto que, esta articulación, permite reparar en ciertos aspectos específicos de su constitución que de otra manera quedarían ignorados. A partir de allí, es posible problematizar algunos de los enunciados más recurrentes referidos a migrantes haitianos y haitianas en Argentina asociados, por ejemplo, a la pobreza, el terremoto ocurrido en 2010 y el modo de entender su vinculación con la diáspora haitiana en el mundo.

Metodológicamente, el trabajo adopta un enfoque cualitativo basado en observación participante y entrevistas en profundidad. Se realizaron 50 entrevistas en profundidad ${ }^{3}$ a un total de 38 personas haitianas (varones y mujeres), 19 en la ciudad de Rosario y 19 en Córdoba, en su mayoría jóvenes entre 18 y 34 años que llegaron al país entre 2007 y agosto de 2018, momento en que se impone un visado de turismo que genera un punto inflexión en el desarrollo de sus redes migratorias y lazos familiares (Trabalón, 2019). Por su parte, la observación participante se produjo, junto a numerosas charlas informales, en diferentes eventos sociales en los que participaron haitianos y haitianas en las ciudades de Córdoba y Rosario, así como también, en espacios

3 Para preservar el anonimato de mis interlocutores, los nombres serán modificados. En los fragmentos de entrevistas la letra " $\mathrm{E}$ " corresponde al entrevistador. 
más reservados como encuentros compartidos o reuniones personales. Este trabajo fue realizado en el transcurso de dos años, desde fines del 2017 hasta fines del 2019, en el marco de mi tesis doctoral en curso sobre control migratorio y fronterizo, trayectorias y procesos de racialización en Argentina.

\section{Los movimientos de haitianos y haitianas hacia la Argentina}

La migración haitiana en Argentina ${ }^{4}$ se inscribe en el marco del impulso y la dimensión que adquieren los movimientos de haitianos y haitianas en la región a partir del 2010, no obstante, es importante destacar que una de las dimensiones más significativas en la reconstrucción de sus trayectorias hacia las ciudades de Córdoba y Rosario fue, precisamente, el señalamiento recurrente sobre la formación de las primeras redes migratorias entre mediados y fines de la década del $2000^{5}$. De hecho, este momento es considerado fundante del tipo de proyecto migratorio con el que la gran mayoría se identifica, es decir, un proyecto familiar con fines de formación universitaria. A partir de allí, los y las jóvenes estiman que, con el pasar de los años, estas redes se van ampliando y consolidando, principalmente, en la ciudad de Rosario, donde el número de haitianos es considerado más representativo. En términos estadísticos, en 2010, la población haitiana en Argentina representaba un número marginal con relación a la población total extranjera, siendo contabilizados solo 309 personas de nacionalidad haitiana al momento de realización del censo en ese año (Debandi, 2017). Más tarde, se observa un aumento progresivo aunque, a diferencia de lo ocurrido en otros países como Ecuador, Brasil y Chile, no se trata de un salto cuantitativo inmediato y relevante con relación a su llegada post-terremoto 2010 (Trabalón, 2018, 2020). Esto puede observarse, por ejemplo, en los ingresos de haitianos y haitianas a la Argentina que, de 2010 a 2014, oscilan entre 1004 a 2166 por año (Del Águila, 2017).

Con relación a las condiciones socio-históricas en las que emergen los movimientos de haitianos en la región, diferentes trabajos refieren a los elevados niveles de desigualdad socio-económica y la recurrente violencia e inestabilidad política, los reiterados "desastres naturales", la crisis política del 2004, la intervención militar de la Misión de Estabilización de las Naciones

4 Los estudios sobre migración haitiana en Argentina son muy escasos. Duffard Evangelista (2014) aborda lo que define como migración forzada de haitianos en Argentina (Buenos Aires) post terremoto 2010 y las dificultades que experimentan con relación a la obtención de derechos y, De Heusch (2016), en su tesis de grado de Antropología, estudia la migración haitiana a partir de la práctica de venta ambulante en la ciudad de Córdoba. También se pueden destacar algunos informes elaborados en colaboración con diferentes organismos internaciones, como Debandi (2017) y Del Aguila (2017).

5 En Córdoba, en particular, hay un antecedente importante de aproximadamente una veintena de haitianos que llega a fines de los sesenta a la ciudad para realizar sus estudios universitarios, muchos de ellos se fueron una vez concluidos sus estudios mientras que otros aún viven en la ciudad. 
Unidas en Haití (MINUSTAH ${ }^{6}$ ) en el mismo año y, como resultado de tal intervención, el brote de cólera de 2010. Sin embargo, se suele coincidir en que uno de los factores decisivos que permitiría comprender la migración haitiana en los países sudamericanos remite al terremoto ocurrido el 12 de enero de 2010, el cual destruyó gran parte de la infraestructura del país (en particular de la capital de Puerto Príncipe) y dejó como saldo más de 200 mil muertos y un millón y medio de desplazados internos, agravando exponencialmente las ya frágiles condiciones sociales de país ${ }^{7}$.

Es importante mencionar que estos hechos tienen lugar en el marco de los procesos históricos recientes de Haití, es decir, en el clima de inestabilidad política, violencia y corrupción generalizada que caracteriza a las últimas décadas, desde el fin de la dictadura duvalierista en el año 1986. En este escenario, la lectura de los procesos migratorios debe ser realizada siempre en el marco de un país cuyas condiciones de vida no dejan de ser continuamente deterioradas, como lo demuestran las estadísticas del país con los niveles de desigualdad más altos de América Latina. Mientras que la tasa de desempleo a principios del 2000 llegaba al 27,2\% (Vil, 2009) en una economía caracterizada por el empleo informal, la pobreza en 2012, según el Banco Mundial, alcanzaba al 68 por ciento de la población. Asimismo, como analiza Gentili (2016), los datos de los últimos años muestran que esta situación está lejos de mejorar, con cerca de diez millones de habitantes, la población de Haití "sobrevive con menos de 1 dólar diario y casi el 80\%, con 2 dólares; el $20 \%$ más rico concentra más del $60 \%$ de los ingresos nacionales, y el 20\% más pobre, apenas el 2\%" (Gentili, 2016, p. 9).

En este contexto, los aspectos estructurales y coyunturales referidos a la historia y situación actual de Haití representan un primer elemento para la comprensión de los proyectos migratorios en las ciudades de Córdoba y Rosario $^{8}$ y, ciertamente, no es posible abordar las trayectorias de haitianos y haitianas sin conocer su vinculación con las condiciones histórico-sociales que caracterizan al país. Sin embargo, se considera necesario realizar dos señalamientos: por un lado, que un análisis de los proyectos migratorios limitado o demasiado centrado en las condiciones de emergencia del país de "origen" puede conducir a perder de vista el entramado global y regional así como los vínculos transnacionales en los que se inscriben estos movimientos.

6 Reconfigurada más tarde como MINUJUSTH (Misión de Apoyo a la Justicia de las Naciones Unidas en Haití) y, en la actualidad, bajo la etiqueta de misión diplomática como BINUH, Oficina Integrada de las Naciones Unidas en Haití.

7 Una primera revisión de antecedentes sobre migración haitiana en la región ha sido realizada en Trabalón, 2018.

8 Un análisis más detallado sobre las razones que llevan a la "elección" de las ciudades de Córdoba y Rosario y, en términos más generales, de Argentina como "destino" ha sido realizado en Trabalón, 2019. 
Por otro lado, que un análisis de las condiciones socio-históricas del país de origen desconectado de la especificidad de los proyectos migratorios puede tener implicancias analíticas importantes $y$, en este sentido, llevar a afirmaciones difícilmente comprobables sobre los proyectos migratorios. Por ejemplo, inferir que porque se trata de un país empobrecido los haitianos que están en Argentina son pobres o que, porque hubo un terremoto en 2010 la migración haitiana hacia la Argentina es una migración forzada por razones humanitarias o que, porque se habla de la diáspora haitiana en el mundo, los haitianos que están en Argentina necesariamente se consideran parte de ésta.

Por ello, sin desconocer las condiciones socio-históricas de Haití, a lo largo del trabajo, se propone problematizar estas miradas situando la conformación de los proyectos migratorios de haitianos hacia las ciudades de Córdoba y Rosario con relación a diferentes escalas, espacios y temporalidades. Así, en lo que sigue, se muestra de qué manera la consideración de las trayectorias sociales permite reconstruir la definición de los proyectos migratorios oponiéndose a aquellas miradas victimizantes que presentan a los sujetos como desprovistos de su capacidad de agencia y, en particular, a aquellas que asocian a los haitianos y haitianas -esencialmente- con la pobreza. No solamente la pobreza y el terremoto en la mayoría de los casos no se hallan vinculados con la especificidad de los proyectos sino que, además, esta misma especificidad permite mostrar que, lejos de la idea de "víctimas", la construcción del proyecto migratorio hacia la Argentina forma parte de estrategias de movilidad construidas generacional, familiar y transnacionalmente en busca de mejores condiciones de vida.

\section{La apuesta por la educación}

Como se explicitaba al inicio, la familia es un componente fundamental en las trayectorias sociales consideradas, de modo que, comprender sus proyectos migratorios es comprender el vínculo entre familias transnacionales, la apuesta por la educación y su condición de jóvenes. A través de este nexo es posible advertir las diferentes maneras en que la edad se constituye en un factor clave de la definición de los proyectos. Esto, de acuerdo con dos dimensiones constitutivas: en primer lugar, una apuesta generacional por la educación como forma de movilidad social y, en segundo lugar, una estrategia de reproducción familiar construida transnacionalmente a través de diferentes redes de parentesco.

Con respecto al primer punto, la apuesta generacional por la educación como forma de movilidad social se presentaba de manera recurrente en los relatos de los y las jóvenes cuando, con el objetivo de reconstruir sus trayectorias, se les preguntaba cómo había surgido Argentina como "destino". En las respuestas que esta pregunta suscitaba, los entrevistados y las 
entrevistadas remarcaban constantemente el valor asignado por los padres a la educación como un lugar común en las razones que explican la conformación del proyecto migratorio hacia la Argentina.

(...) ellos no sabían leer. Existe eso de los padres, porque hay mucha gente allá que no sabe leer. Ellos dicen 'yo no tuve esa oportunidad' o 'esa suerte' como dicen allá; 'a mi hijo eso no le va a pasar'. Entonces hacen mucho esfuerzo. (Joseph, 32 años, reside en Argentina desde 2011)

(...) hay un montón de familias que los padres no saben leer ni escribir pero sus hijos son ingenieros, son médicos, son tal y tal cosa. Cuando pasa eso, tu mamá o tu papá que no quiere que tengas el mismo futuro que él lo que hace te empuja a llegar, te empuja. Ahora, tu éxito es su sueño, él no tuvo suerte de ser médico pero sueña en ser médico por vos, mi hijo es médico, yo me siento médico. (Robert, 29 años, reside en Argentina desde 2008)

Estos fragmentos, que fácilmente pueden hacerse extensivos a lo expresado en la mayoría de los relatos, permiten señalar entonces que, aunque las trayectorias familiares son diversas, existe un trasfondo común asentado en la búsqueda de movilidad social a través de la apuesta por la educación de estos y estas jóvenes. Hay una distinción entre una mayoría que es primera generación en acceder a los estudios universitarios y, en varios casos, a la escolarización y, aquellos que son segunda generación de estudiantes universitarios con padre o madre profesional (raramente ambos). De acuerdo con estas diferencias, podría decirse que se remarcaba, en mayor o menor medida, el carácter "sacrificado" de las trayectorias laborales de sus padres puesto que, muchas veces remitían a trabajos poco calificados y realizados en condiciones precarias. Sin embargo, en general, se coincidía en destacar a través de palabras como "orgullo" y "agradecimiento" lo que consideran que ha sido un "esfuerzo" de sus padres para que ellos pudieran acceder a la educación y llegar a la instancia de formación universitaria.

Este reconocimiento a través de las ideas de "sacrificio" y "esfuerzo" de los padres, además, cobra sentido en un contexto particular si se tiene en cuenta que Haití, como señala Gentili (2016), a pesar de ser el primer país de América Latina que tuvo una ley de escolaridad obligatoria no establece ni garantiza en términos normativos la gratuidad de la educación. Por el contrario, el país más empobrecido de América, "es el que tiene el sistema escolar más privatizado en todo el continente, con el 90\% de las escuelas bajo el comando de las Iglesias, ONG y pequeños empresarios, que albergan a más del $80 \%$ de la población escolar" (Gentili, 2016, p. 6). Por lo tanto, señala este autor, la escolarización de los y las niños y jóvenes pasa a recaer fundamentalmente sobre las familias haitianas.

En este sentido, sean de primera o segunda generación, no se observan grandes cambios con relación al modo en que los y las jóvenes definen sus proyectos migratorios, en los que se destaca el valor asignado por los padres 
a la educación como matriz fundante de los mismos ${ }^{9}$. Así, los fragmentos expuestos remiten a la descripción más recurrente de los proyectos migratorios, esto es, un logro que se presenta como resultado del trabajo de los padres y que es percibido por los hijos/as, con mayor o menor énfasis, en términos de un "esfuerzo" que debe ser valorado y sobre el cual hay que estar "agradecido" pero también, por esto mismo, como una circunstancia a partir de la cual se desprenden una serie de obligaciones y deberes que en tanto jóvenes le corresponden dentro el núcleo familiar.

De acuerdo con esto, el vínculo entre migración y juventud se presenta en la definición de estos proyectos a través de mandatos familiares configurados en base a una apuesta por la educación construida generacionalmente. Como sostiene Martin-Criado (2005), la juventud en términos sociológicos no se refiere a la edad biológica sino a una edad social que, como clase de edad, remite a divisiones sociales que adquieren un carácter performativo. Es decir, cada clase de edad (infancia, juventud, vejez, etc.) supone "una forma de pensamiento y comportamiento socialmente definida y los sujetos tienden a adecuarse a la definición social de la categoría en la que se hallan incluidos" (p. 88). Siguiendo el planteo de Bourdieu y Manheim, Martín-Criado (2009) señala que estas divisiones sociales operan diferente según los diversos grupos sociales, por lo que, no solo dependen de las condiciones materiales y sociales de vida sino también y, sobre todo, de las estrategias de reproducción social. En otras palabras, las divisiones no son definidas externamente sino al interior de grupos sociales específicos y, por ello, la división de las clases de edad difiere según las posiciones ocupadas en el espacio sociológicamente construido de las familias. De este modo, en la mayoría de los casos, el proyecto migratorio emerge como producto de este mandato familiar asociado a una movilidad social que evalúa el estudio en el extranjero como un recurso valioso en el marco de sus estrategias de reproducción familiar, entendidas más que como intenciones conscientes y a largo plazo, como el conjunto de acciones tendientes a aumentar o a conservar su patrimonio, manteniendo o mejorando su posición en la estructura de las posiciones de clase (Bourdieu, 2011).

Ser joven en el seno de estas familias, significa asumir una serie de deberes y obligaciones al finalizar los estudios secundarios. En primer lugar, con respecto a una posibilidad siempre latente de hacerlo en otro país por el valor diferencial que se le otorga a los títulos universitarios obtenidos en el

\footnotetext{
9 Aquí es importante subrayar que, si bien esta distinción entre diversos orígenes sociales no cambia sustancialmente en lo que se refiere a la definición de los proyectos migratorios, resulta relevante señalarla para entender los procesos de desclasamiento que atraviesan estas trayectorias en Argentina. Esto teniendo en cuenta, además, otros factores como los vínculos transnacionales, las políticas migratorias, los procesos de racialización y las dinámicas locales de cada ciudad, elementos que, por cuestiones de extensión, no podrán ser abordados en este trabajo.
} 
extranjero ${ }^{10}$, de manera que, la misma acción de migrar puede ser interpretada dentro de este mandato social y familiar. En efecto, el valor de la educación asignado por estas familias se comprende a través del vínculo entre juventud y proyección al futuro y, la configuración de este vínculo como impulso para el desarrollo de estrategias de movilidad social que comprenden a todo el grupo familiar. Como lo expresan dos jóvenes "desde siempre tenía la idea de irme de Haití porque tus padres desde chico te dicen 'en Haití no hay futuro'"11 o, "como siendo joven no te sientes bien, no hay una buena vida, entonces buscas irte"12. De este modo, para gran parte de estos y estas jóvenes y sus familias, esta proyección al futuro solo es posible a través de la migración:

Sí, uno si viera esa situación desde su país, los jóvenes miran el gobierno, miran la sociedad, ven que la única opción que lamentablemente tenemos es viajar. El haitiano siempre quiere salir adelante: aunque no es el trabajo que uno quería, pero la idea es siempre así: "mientras no estoy en Haití, yo estaré bien". Ese es el pensamiento de muchos. (Evens, reside en Argentina desde 2009)

En segundo lugar, ser joven también tiene que ver, de acuerdo a las trayectorias sociales que apuestan generacionalmente por la educación, con la obligación de realizar una formación universitaria en carreras predeterminadas entre las que se destaca la de Medicina.

En Puerto Príncipe estudié dos años dentista, odontología digamos. No me gustó, me di cuenta que no era para mí. Pero mi mamá siempre tenía esa idea que yo fuera médica. Lo que fuera, pero que sea médica. Pero bueno, no pude estudiar allá porque es muy complicado. Hay una sola Universidad Nacional para todo el país, así que imaginate que vienen chicos de todos lados. Toman cien personas en cada Facultad. (Lyne, reside en Argentina desde 2005)

(...) yo quería ser médica. Porque viste que, no sé por qué, nosotros, los haitianos, tenemos como una meta de ser médico, abogado o enfermero, algo así. Y yo quería ser médica. (Pamela, reside en Argentina desde 2017)

Y después de terminar mi estudio, bueno, intentaba muchas cosas, porque allá la familia un poco te guía muchas veces. A ver, no digo que está mal, pero es costumbre como ya la familia sabe dónde te quieren meter. Bueno, "Te quiero hacer médico", "Te quiero hacer abogado", "Te quiero hacer ingeniero", pero muchas veces, vos que estás estudiando, capaz te acostumbras más en Agronomía. Y eso pasa en el 90 por ciento de las familias. Y muchas veces, cuando uno viaja, a veces la familia manda. (Claude, reside en Argentina desde 2008)

En este marco, el mandato profesional se entiende a partir del significado que cobra para los y las jóvenes el proyecto migratorio como inversión familiar a largo plazo que se traduce en "destino" bajo la forma de compromiso familiar.

\footnotetext{
${ }_{10} \mathrm{Al}$ mismo tiempo, los profesionales formados en el sistema universitario de Haití se dirigen a países como República Dominicana y, cuando es posible, a Canadá, los Estados Unidos o Francia, siendo casi el $85 \%$ de los haitianos con nivel superior de educación el porcentaje de los que han salido del país durante los últimos años (Gentili, 2016).

11 Alí, reside en Argentina desde 2014.

12 Fátima, reside en Argentina desde 2016.
} 
Esto es así en la medida que la gran mayoría de los proyectos se construyen transnacionalmente a través del apoyo de diversas redes de parentesco y los y las jóvenes son conscientes de esta apuesta familiar y su papel dentro de ella.

Es toda una carga para nosotros, es así, el haitiano vive así porque sino no estaríamos acá. Cuando uno viene acá en la Argentina y se queda sin plata, puede llamar al tío, al papá, a la mamá, hay como todo un respaldo y cuando tu llegas tenés que esperar que el primito te va a llamar, el hermanito te llama, el vecinito también te puede llamar porque toda esa contribución para llegues a tu destino es plata prestada, te ayudan a llegar vos, tenes que ayudar a los otros a llegar también, como una cadena porque si todos llegamos no va a ser un peso para vos, te puedo ser peso durante cinco, seis años mientras me estoy preparando pero sino nunca me preparo siempre voy a ser un peso, si me preparo, al contrario, si me preparo voy a ser un peso durante cinco o seis años y después podemos ser colegas, podemos ser parte de un proyecto para cambiar las cosas. (Robert, 28 años, reside en Argentina desde 2008)

Empezamos a hablar sobre el tema y ahí ella me contó que acá el estudio es gratis y todo eso, pero que lo que más caro es el pasaje. Y una vez que llegas acá, tu familia tiene que ayudarte también por el tema de vivienda, de comida y todo eso. Y bueno, de ahí hablé de eso con mi familia y decidieron ayudarme. E: ¿Y tu familia a qué se dedica? S: Tengo, bueno, tíos que viven en Estados Unidos, porque mi mamá ya no trabajaba, no me podía comprar el boleto. Así que, mis tíos y tías que están allá, o sea, pusieron plata juntos y me compraron el boleto. (Stéphane, 26 años, reside en Argentina desde 2013)

En la medida que los proyectos son resultado de una inversión familiar y esta inversión se configura a través de las redes de familiares en diferentes países del mundo, los vínculos transnacionales son considerados como la segunda dimensión constitutiva de los proyectos.

\section{Familias transnacionales}

En general, los proyectos migratorios de haitianos y haitianas que residen en Córdoba y Rosario se llevan adelante en conexión con la realización simultánea o pasada de experiencias migrantes de distintos miembros de la familia. De este modo, podría decirse que cada proyecto tiene lugar en un espacio estructurado de posiciones que se genera en el marco de relaciones de poder entre actores e instituciones de dos o más países que se vinculan a través de las prácticas de los migrantes (Suárez Navaz, 2006). Por lo tanto, esta primera parte de la reconstrucción de las trayectorias sociales permite mostrar la relación existente entre la conformación de los proyectos migratorios de estos y estas jóvenes y los recorridos migrantes de distintos miembros de la familia como condición de posibilidad para llevarlos adelante. Como resultado de estas apuestas familiares, los vínculos transnacionales son diversos ya que no solo son importantes en términos económicos para impulsar el proyecto sino también para sostenerlo a lo largo del tiempo. 
Haciendo foco en el momento de definición, según los relatos, los vínculos de parentesco han sido una pieza clave en la concreción de los proyectos en la medida que son los familiares quienes deciden invertir en ellos o ellas aportando económicamente para los pasajes pero también para la estadía de los primeros meses, algunos años o durante toda la carrera. De acuerdo con lo manifestado por los y las jóvenes, el aporte a los proyectos puede provenir de distintos miembros de la familia. Sin embargo, hay una trayectoria que se repite con mayor frecuencia, aquella que refiere a los casos en que la madre o el padre ha migrado en algún momento de su vida, principalmente a Estados Unidos, Canadá y Francia pero también a otros países del Caribe como Cuba, Las Bahamas y República Dominicana, con el objetivo de trabajar para mantener a sus familias en Haití y que ellos y ellas puedan escolarizarse. Esto permite reforzar la afirmación según la cual el proyecto migratorio es una apuesta generacional de los padres que tiene como finalidad, en principio, lograr que sus hijos o hijas accedan a la educación en Haití hasta finalizar el nivel secundario y, luego, evaluar las posibilidades de acceso a la educación universitaria en Haití o, preferentemente, en países extranjeros por el peso diferencial que se estima poseen sus títulos.

Asimismo, como ya se mencionó, buena parte de los haitianos y las haitianas que se encuentran en las ciudades de Córdoba y Rosario tienen un padre o madre y/o tíos, tías, primos, primas que son migrantes y aportan económicamente a sus proyectos. Así como también, muchas veces, sus hermanos o hermanas se encuentran realizando sus estudios en otros países o, habiéndose recibido como profesionales trabajando en otros países o, en el caso de aquellos hermanos o hermanas más jóvenes, con el proyecto realizar sus estudios en el exterior una vez que finalicen sus estudios secundarios en Haití. En este punto es importante resaltar que la conexión entre las trayectorias migrantes familiares y los proyectos migratorios analizados se encuentra mediado por lo que ha sido prolíficamente estudiado como la "diáspora haitiana". En este trabajo interesa recuperar esta categoría especialmente con relación al sentido social que se construye desde la perspectiva de los y las jóvenes. Como sostiene Joseph (2015),

(...) tanto fuera como dentro de Haití, el término diáspora es una categoría organizadora en el mundo, ya que designa personas, califica objetos, dinero, casas y acciones. El término diáspora se usa para designar compatriotas que viven en el extranjero, pero que regresan temporalmente a Haití y luego regresan al extranjero: diáspora ki jan o ye? (diáspora, cómo estás?). El campo semántico y polisémico del término está articulado por tres verbos asociados con la diáspora: residir en el extranjero, regresar a Haití y volver al extranjero. (Joseph, 2015, p. 53 , traducción propia)

En esta línea, se sostiene que, aunque la categoría de diáspora sirve para comprender la definición de los proyectos migratorios por su vinculación 
con otras redes migratorias construidas históricamente en otros países, lo más importante aquí es que habilita al señalamiento de principios de distinción fundamentales que dan cuenta de la especificidad de estas trayectorias. Esto, con relación a las tensiones que se producen entre la consideración de la "diáspora" como categoría analítica y su uso como categoría nativa a través de los diferentes sentidos sociales que se le atribuye al término. De este modo, se coincide con el planteo de Joseph (2015), quien a partir de su trabajo sobre haitianos en movimiento por Brasil, Suriname y Guyana Francesa, señala que las nuevas formas de movilidad haitiana a nivel internacional y, en particular, en la región sudamericana, habilitan nuevos abordajes con relación a la polisemia del término diáspora.

En este caso de estudio, "ser diáspora" es una definición social construida en origen que pasa a ser resignificada a partir de las tensiones que genera con su propia experiencia migrante. Para la mayoría el término diáspora está asociado con la capacidad financiera del migrante que vive en el exterior, va de visita a Haití y se vuelve a ir y, por ello, también se encuentra vinculada principalmente con la migración más tradicional hacia Estados Unidos, Canadá y Francia y, algunos casos, del Caribe. Desde la percepción de muchos y muchas jóvenes, uno de los sostenes más importantes de la economía haitiana lo componen las remesas de los migrantes, cuestión que se traduce simbólicamente en un principio de distinción que determina quién es diáspora y quién no lo es. Por ello, desde su condición de jóvenes con proyectos educativos universitarios, en algunos casos todavía sin haber ingresado al mercado laboral y, los que lo han hecho, por lo general, en mercados laborales precarizados (con la excepción de una minoría de profesionales formados mayormente en Argentina), se entiende que la mayoría de ellos no se consideren parte de la diáspora sino en "deuda" con esta ${ }^{13}$. En todo caso, ser "un diáspora" es algo que se espera a largo plazo en la medida que, una vez recibidos, asuman otra posición en el esquema de mandatos, deberes y obligaciones familiares, "si yo estoy estudiando acá, ¿cómo voy a ser diáspora? No, diáspora es otra cosa, en otro momento quizás, ahora no lo soy"14.

Teniendo en cuenta, entonces, el lugar ocupado por la "diáspora" en la definición del proyecto migratorio, a su vez, entendido como una apuesta generacional de movilidad social a través de la educación en el marco de las estrategias de reproducción familiar y los roles específicos asignados a los y las jóvenes en éste, se comprende que la misma decisión de emprender el viaje hacia la Argentina sea resultado de, en varios casos, una negociación del o la

\footnotetext{
${ }^{13}$ Otro sentido predominante sobre ser diáspora se refiere al hecho de vivir en el extranjero, independientemente de la capacidad financiera del migrante.

${ }^{14}$ Joseph, reside en Argentina desde 2011.
} 
joven con su familia y, en muchos otros, una determinación de la familia sin participación de los y las jóvenes en el proceso de toma de decisión.

Y tenía en ese momento como 19 años. Y empecé a buscar, a buscar... hasta que encuentro un amigo de la familia que ya estaba acá, que llevaba como tres años acá. Y contacté con esta persona y me contó cómo es la realidad de acá, las ventajas sobre todo de la universidad pública y gratuita, todo eso (...). Y les conté a mis padres y les convencí. Así que, empezaron a hacer todos los trámites para que yo pueda venir acá. (Pierre, 23 años, reside en Argentina desde 2016)

E: ¿Y cómo surge la idea de venirte para Argentina? S: Y, mi hermana, que está viviendo en Francia, tiene una amiga acá en Rosario. Y estaban como reflexionando a dónde me mandaban para el estudio universitario, porque allá en mi país es un poco complicado el tema de la universidad. De costo y de la inseguridad. Así que, bueno, estaban reflexionando entre ellos... Así que, llegaron a un resultado con mis padres de mandarme acá con una amiga que estaba viviendo por acá. E: ¿Y vos estabas cuando ellos hablaban sobre eso? S: Yo no. Me dijeron, "Bueno, sabés, el mes que viene te vamos a comprar el boleto para ir allá, ya hicimos todos los trámites, todo". Les dije, "Sí, bueno", ya está. Así que, estoy acá en Argentina. (Samuel, 20 años, reside en Argentina desde 2018)

(...) la madre de mis primos, habló con mi mamá también sobre que podía venir a estudiar acá Medicina. Pero cuando mi mamá me dijo eso, le dije, “iPero mamá, no sé nada de español! Pero el año que entró mi tío no tenía nada que decir, porque las facultades de allá estaban en problemas, privadas y públicas. Y por eso mi papá me dijo, "No tenés nada que decidir, te vas a ir y hacer la carrera allá". E: O sea que decidieron tus padres? .N: Sí!! (Natacha, 20 años, reside en Argentina desde 2017)

Reparar en la especificidad de los proyectos permite comprender cómo estos y estas jóvenes, más allá de la heterogeneidad social que les caracteriza, pertenecen a un modo de generación (Sayad, 2010) particular asociado a determinadas condiciones sociales de existencia. Estas condiciones sociales remiten a los aspectos estructurales y coyunturales de la historia de Haití y de la migración haitiana en el mundo, de las cuales resultan los mandatos familiares vinculados al desarrollo de los proyectos así como también la función asignada en cada uno de ellos y ellas en tanto jóvenes en un contexto histórico-social que hace casi impensable su proyección al futuro en Haití. Al mismo tiempo, aunque muchas familias estén compuestas por padre o madre migrantes, en general se trata de generaciones distintas de migrantes, esto es, han sido generados desde posiciones sociales distintas y, por lo tanto, son diferentes modos de generación. Como señala Martín-Criado (1998), “cuando cambian las condiciones de reproducción de los grupos sociales y, por lo tanto, las condiciones sociales y materiales de producción de nuevos miembros, es cuando se producen diferencias de generación: los nuevos miembros son generados de manera distinta" (Martín-Criado, 1998, p. 83). De tal forma, las estrategias de reproducción son las que permiten explicar los cambios en los modos de reproducción que está en el origen de la aparición de generaciones distintas (Mauger, 2013). 
En este caso, el modo de generación de estos y estas jóvenes puede pensarse en el marco del proceso de movilidad social ascendente que venía atravesando su familia, al menos hasta el momento de definición de sus proyectos migratorios. En este sentido, se entiende también que la pregunta por el terremoto produzca malestar por considerar que la imagen construida sobre el haitiano asociado al terremoto y, por lo tanto, a la pobreza, no se corresponde ni con sus proyectos ni con su posición social. Así, si bien algunos haitianos y haitianas que llegan en los años inmediatos al terremoto, reconocen que sus proyectos migratorios se relacionan, potencian o cobran mayor impulso a partir de este evento, en general, para aquellos y aquellas jóvenes con los que se ha tenido oportunidad de dialogar y compartir diferentes espacios, la idea de estudiar en el extranjero así como la posibilidad de migrar del país es un elemento pre-existente al terremoto, en otros muy distante y, para muchos, sin relación directa con sus proyectos e "identidad". Como señalaba una joven, "no es como dice la mayoría de la gente acá, que uno viene por el terremoto. El terremoto fue un evento que pasó en Haití, pero no es que identifica o es la identidad de todos los haitianos"15.

\section{Conclusiones}

El análisis realizado posibilitó dar cuenta cómo la edad resulta un factor estructurante en la definición de los proyectos migratorios que, a su vez, son entendidos en el marco de las estrategias de reproducción familiar. Como tales, se encuentran atravesados por las concepciones existentes al interior de estas familias sobre lo que significa ser joven y los deberes y obligaciones que esta figura incluye. En este caso, las familias consideran la educación como un valor central en tanto estrategia de movilidad social y reproducción familiar. Esta estrategia es construida generacionalmente e involucra trayectorias migrantes de diferentes tipos que dan cuenta de una existencia previa de vínculos transnacionales que conectan el proyecto migratorio con redes de relaciones familiares que se han establecido a lo largo del tiempo y en conexión con diversos lugares/países. En tal sentido, las familias son transnacionales antes que los y las jóvenes emprendan sus proyectos migratorios y lo que se produce en todo caso a partir del proyecto migratorio hacia la Argentina es una reconfiguración de esos vínculos transnacionales y, por lo tanto, en los modos en que se conforman las relaciones al interior de cada familia. La diáspora como categoría analítica sirve para pensar estos vínculos previos a través de los cuales se gesta el proyecto migratorio hacia la Argentina pero en este país adquiere para muchos y muchas un sentido diferente.

15 Pamela, reside en Argentina desde 2012. 
Por consiguiente, la definición de los proyectos migratorios forma parte de trayectorias sociales más amplias consideradas no sólo a partir de las relaciones entre las estructuras familiares y las estructuras de la migración a lo largo de distintas generaciones sino también en un marco espacial y temporal todavía más amplio en el que las estrategias de movilidad de los sujetos son entendidas a partir de diferentes conexiones transnacionales. A pesar de las dificultades que aparecerán en la concreción de estos proyectos en las ciudades de Córdoba y Rosario, el análisis muestra la importancia de considerarlos en el marco de la heterogeneidad social que los caracteriza, evitando miradas reduccionistas y/o victimizantes que obturan la compresión de las estrategias y formas de negociación en diferentes espacios, momentos y situaciones a través de los cuales, estas "nuevas" juventudes en el país, intentan sostener sus proyectos y así, seguir en movimiento.

\section{Referencias bibliográficas}

AVALLONE, Genaro; SANTAMARÍA, Enrique (coords.). Abdelmalek Sayad: una lectura crítica. Migraciones, saberes y luchas (sociales y culturales). Madrid: Dado Ediciones, 2018.

BEHERAN, Mariana. Niños, niñas y jóvenes bolivianos y bolivianas en la ciudad de Buenos Aires. Escolaridad y experiencias formativas en el ámbito familiar. Estudios Migratorios Latinoamericanos, v. 22, n. 67, p. 375-396, 2009.

BOURDIEU, Pierre. Cosas dichas. Buenos Aires: Gedisa, 1987.

BOURDIEU, Pierre. Las estrategias de reproducción social. Buenos Aires: Siglo XXI, 2011.

BOURDIEU, Pierre; WACQUANT, Loïc. The organic ethnologist of Algerian migration. Ethnography, v. 1, n. 2, p. 173-182, 2000.

DEBANDI, Natalia. Diagnóstico regional sobre migración haitiana. Buenos Aires: OIM/IPPDH, 2017.

DE HEUSCH, Félicien. "Amo a mi país, sigo hablando en creole, pero tomo fernet con coca y como el asado": Una etnografía de los migrantes haitianos y de la venta de "bijouteria" en la ciudad de Córdoba, Argentina. Tesis de Licenciatura. Universidad Nacional de Córdoba, Córdoba, 2016.

DEL ÁGUILA, Álvaro. Estudio exploratorio sobre las trayectorias socioeducativas y socio-laborales de migrantes haitianos, senegaleses y ucranianos en la ciudad de Buenos Aires. Buenos Aires: OIM/FCCAM, 2017.

DOMENECH, Eduardo. Inmigración, política (s) y "pensamiento de Estado": la mirada crítica heterodoxa de Abdelmalek Sayad. Conferencia, San Pablo, Octubre de 2018. Disponible en: <https://www.academia.edu/38990141/ Inmigraci\%C3\%B3n_pol\%C3\%ADtica_s_y_pensamiento_de_Estado_la_mirada_ cr\%C3\%ADtica_heterodoxa_de_Abdelmalek_Sayad>. 
DUFFARD, Irene. Del Caribe haitiano a la Argentina: Trayectorias de cuerpos en movilidad humana pos terremoto 2010. Buenos Aires: CLACSO, 2016. Disponible en: < http://biblioteca.clacso.edu.ar/clacso/pobreza/20160307040904/Duffard. $\operatorname{pdf}>$.

FELDMAN-BIANCO, Bela. Desarrollos de la perspectiva transnacional: migración, ciudad y economía política. Alteridades, v. 25, n. 50, p. 13-26, 2015. Disponible en: <http://www.scielo.org.mx/scielo.php?pid=S0188$70172015000200002 \&$ script $=$ sci_arttext\&tlng $=$ pt $>$.

FELDMAN-BIANCO, Bela. Anthropology and ethnography: the transnational perspective on migration and beyond. Etnográfica. Revista do Centro em Rede de Investigação em Antropologia, v. 22, n. 1, p. 195-215, 2018. Disponible en: $<$ https://journals.openedition.org/etnografica/5203>.

GARCÍA BORREGO, Ignacio. Jóvenes migrantes y sociedades en tránsito. In: LÓPEZ SALA, Ana; CACHÓN RODRIGUEZ, Lorenzo (coords.). Juventud e inmigración. Desafíos para la participación y la integración. Caracas: Dirección General de Juventud de la Consejería de Empleo y Asuntos Sociales del Gobierno de Canarias, 2007, p. 158-171.

GAVAZZO, Natalia. No soy de aquí, ni soy de allá.... Alterización y categorías de identificación en la generación de los hijos de inmigrantes bolivianos y paraguayos en Buenos Aires. Claroscuro. Revista del Centro de Estudios sobre la Diversidad Cultural, n. 12, p. 73-95, 2013. Disponible en: < http://rephip.unr. edu.ar/handle/2133/12708>.

GAVAZZO, Natalia. Jóvenes migrantes e hijos de inmigrantes latinoamericanos en Buenos Aires: una generación en movimiento. Confluenze. Rivista di Studi Iberoamericani, v. 10, n. 1, p. 131-165, 2018. Disponible en: < https:// confluenze.unibo.it/article/view/8298>.

GENTILI, Pablo. La educación en Haití: el abandono al caos. Buenos Aires: CLACSO, 2016.

GERBAUDO SUÁREZ, Débora. Juventudes "Latinoamericanas" en Buenos Aires. Luchas migrantes y configuraciones trasnacionales de lo local. Revista Argumentos, v. 15, n. 1, p. 213-234, 2019.

GIL ARAUJO, Sandra. Una sociología (de las migraciones) para la resistencia. Empiria. Revista de metodología de ciencias sociales, n. 19, p. 235-273, 2010. Disponible en: < http://revistas.uned.es/index.php/empiria/article/viewFile/2025/1905>.

GIL ARAUJO, Sandra; PEDONE, Claudia. Familias migrantes y estados: vínculos entre Europa y América Latina. Papeles del CEIC, International Journal on Collective Identity Research, v. 2, n. 1, p. 1-24, 2014. Disponible en: <http:// dx.doi.org/10.1387/pceic.13020>.

GLICK-SCHILLER, Nina. A Global Perspective on Migration and Development. In: GLICK-SCHILLER, Nina; FAIST, Thomas (eds.). Migration, Development and Transnationalization. Nueva York-Oxford: Berghan Books, 2010, p. 22-62. 
GROISMAN, Lucía. Jóvenes migrantes costureros: experiencias formativas, relaciones generacionales y subjetivación política en la Ciudad de Buenos Aires. ODISEA. Revista de Estudios Migratorios, n. 6, p. 166-199, 2019. Disponible en: <https:// publicaciones.sociales.uba.ar/index.php/odisea/article/view/4686>.

JIMENEZ ZUNINO, Cecilia. Sayad en uso: trayectorias y proyectos migratorios como herramientas de análisis. In: AVALLONE, Genaro; SANTAMARÍA, Enrique (coords.). Abdelmalek Sayad: una lectura crítica, Migraciones, saberes y luchas (sociales y culturales). Madrid: Dado Ediciones, 2018, p. 75-92.

JOSEPH, Handerson. Diaspora. Sentidos sociais e mobilidades haitianas. Horizontes Antropológicos, v. 21, n. 43, p. 51-78, 2015. Disponible en: < http://www.scielo. br/scielo.php?pid=S0104-71832015000100051\&script=sci_arttext $>$.

LENOIR, Remi. Objeto sociológico y problema social. In: CHAMPAGNE, Patrick; LENOIR, Remi; MERLLIÉ, Dominique; PINTO, Louis. Iniciación a la práctica sociológica. Madrid: Siglo 21, 1993, p. 57-102.

LEVITT, Peggy; GLICK-SCHILLER, Nina. Perspectivas internacionales sobre la migración: conceptualizar la simultaneidad. Revista Migración y Desarrollo, n. 3, p. 60-91, 2004. Disponible en: < https://mww.redalyc.org/articulo.oa?id=66000305>.

MAGGI, Florencia; HENDEL, Verónica. Experiencias escolares desde el prisma del desplazamiento. Revista Temas de Antropología y Migración, n. 11, En prensa, ISSN: 1853-354.

MARTÍN-CRIADO, Enrique. Producir la juventud: crítica de la sociología de la juventud. Madrid: Istmo, 1998.

MARTÍN-CRIADO, Enrique. La construcción de los problemas juveniles. Nómadas (Col), n. 23, p. 86-93, 2005. Disponible en: < https://www.redalyc.org/articulo. oa?id=105116741010>.

MARTÍN-CRIADO, Enrique. Generaciones/clases de edad. In: REYES, Román (dir.). Diccionario Crítico de Ciencias Sociales: Terminología Científico-Social. MadridMéxico: Plaza y Valdés, 2009. Disponible en: < https://webs.ucm.es/info/ eurotheo/diccionario/G/generaciones.htm>.

MAUGER, Gérard. "Modos de generación" de las "generaciones sociales". Sociología Histórica, n. 2, p. 131-151, 2013. Disponible en: < https://revistas. um.es/sh/article/view/188951>.

MIRANDA, Ana; CRAVINO, María; MARTÍ GARRO, Santiago. Transiciones juveniles de migrantes paraguayos/as en la argentina: condiciones de vida y vigencia de las redes. Última década, v. 20, n. 37, p. 11-39, 2012. Disponible en: < http:// dx.doi.org/10.4067/S0718-22362012000200002>.

NOVARO, Gabriela. Migración boliviana, discursos civilizatorios y experiencias educativas en Argentina. Nómadas, v. 45, n. 10, p. 105-121, 2016. Disponible en: < https://ri.conicet.gov.ar/handle/11336/50535>.

NOVARO, Gabriela. Migración boliviana y escuela secundaria en Argentina: reflexiones en clave intercultural. Autoctonía. Revista de Ciencias Sociales e Historia, v. 3, n. 2, p. 111-131, 2019. Disponible en: < https://doi.org/10.23854/ autoc.v3i2.133>. 
ROSAS, Carolina Alejandra. "Yo pensaba que era como en las películas..." Reflexiones en torno a los jóvenes latinoamericanos y las migraciones internacionales. Tendencias en foco, n. 27, p. 1-20, 2014. Disponible en: < https://ri.conicet.gov. ar/handle/11336/35463>.

SAYAD, Abdelmalek. La doble ausencia: De las ilusiones del emigrado a los padecimientos del inmigrado. Barcelona: Anthropos, 2010.

SUÁREZ NAVAZ, Liliana. Un nuevo actor migratorio: jóvenes, rutas y ritos juveniles transnacionales. In: OLMOS, Francisco Checa; ARJONA, Angeles; OLMOS, Juan Carlos Checa (eds.). Menores tras la frontera: otra inmigración que aguarda. Barcelona: Icarias, 2006, p. 17-50.

TRABALÓN, Carina. Violencia estatal, control fronterizo y racialización: experiencias de haitianos y haitianas en aeropuertos de Argentina. Revista Historia y Sociedad, n. 39, p. 155-183, 2020. DOI:10.15446/hys.n39.82972.

TRABALÓN, Carina. Estrategias de movilidad, visados y fronteras: Trayectorias de haitianos y haitianas hacia la Argentina. Revista Estudios Fronterizos, v. 20, n. 4, p. 1-23, 2019. Disponible en: <https://doi.org/10.21670/ref.1918039>.

TRABALÓN, Carina. Política de visado y regulación de las fronteras. Un análisis desde la movilidad de haitianos en Sudamérica. Polis (Santiago), Revista Latinoamericana, v. 17, n. 51, p. 163-186, 2018. DOI: 10.32735/S07186568/2018-N51-1354.

TRPIN, Verónica. Identidades en movimiento: familias chilenas en la fruticultura del Alto Valle de Río Negro, Argentina. Cadernos Pagu, n. 29, p. 227-255, 2007.

VIL, Enel. Pobreza y desigualdad en Haití: un análisis de sus determinantes. México: FLACSO, 2009.

ZENKLUSEN, Denise. «Quiero seguir estudiando para ser alguien»: análisis de trayectorias educativas de jóvenes peruanos en Argentina. RLCSNJ, v. 18, n. 2, p. 1-27, 2020. Disponible en: <https://doi.org/10.11600/1692715x.18203>. 